\title{
Sleep maintenance, spindling excessive beta and impulse control: an RDoC arousal and regulatory systems approach?
}

\author{
Martijn Arns ${ }^{1,2^{*}}$, Ron J Swatzyna ${ }^{3}$, Jay Gunkelman ${ }^{4}$ and Sebastian Olbrich ${ }^{5}$
}

* Correspondence:
martijn@brainclinics.com
${ }^{1}$ Department of Experimental
Psychology, Utrecht University,
Utrecht, The Netherlands
${ }^{2}$ Research Institute Brainclinics,
Bijleveldsingel 34, Nijmegen
6524 AD, The Netherlands
Full list of author information is
available at the end of the article

* Correspondence: martijn@brainclinics.com Psychology, Utrecht University, Utrecht, The Netherlands available at the end of the article

\begin{abstract}
Background: In 2009 the United States National Institute of Mental Health (NIMH) introduced the Research Domain Criteria (RDoC) project, which intends to explicate fundamental bio-behavioral dimensions that cut across heterogeneous disorder categories in psychiatry. One major research domain is defined by arousal and regulatory systems.

Methods: In this study we aimed to investigate the relation between arousal systems (EEG-beta phenotypes also referred to as spindling excessive beta (SEB), beta spindles or sub-vigil beta) and the behavioral dimensions: insomnia, impulsivity/ hyperactivity and attention. This analysis is conducted within a large and heterogeneous outpatient psychiatric population, in order to verify if EEG-beta phenotypes are an objective neurophysiological marker for psychopathological properties shared across psychiatric disorders.
\end{abstract}

Results: SEBs had an occurrence between 0-10.8\% with a maximum occurrence at frontal and central locations, with similar topography for the heterogeneous sample as well as a more homogenous ADHD subgroup. Patients with frontal SEBs only, had significantly higher impulsivity/hyperactivity (specifically on impulse control items) and insomnia complaints with medium effect sizes.

Conclusions: Item level and mediation analysis revealed that sleep maintenance problems explained both frontal SEB EEG patterns (in line with SEB as a sub-vigil or hypoarousal EEG pattern) as well as the impulse control problems. These data thus suggest that frontal SEB might be regarded as a state marker caused by sleep maintenance problems, with concurrent impulse control problems. However, future longitudinal studies should investigate this state-trait issue further and replicate these findings Also studies manipulating SEB by for example neurofeedback and measuring consequent changes in sleep and impulse control could shed further light on this issue.

Keywords: QEEG, Beta Spindles, Neurofeedback, Insomnia, Impulse control, Impulsivity, ADHD, Research domain criteria, Vigilance, Arousal

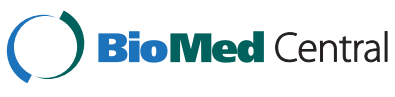

(c) 2015 Arns et al.; licensee BioMed Central. This is an Open Access article distributed under the terms of the Creative Commons Attribution License (http://creativecommons.org/licenses/by/4.0), which permits unrestricted use, distribution, and reproduction in any medium, provided the original work is properly credited. The Creative Commons Public Domain Dedication waiver (http://creativecommons.org/publicdomain/zero/1.0/) applies to the data made available in this article, unless otherwise stated. 


\section{Background}

In 2009 the United States National Institute of Mental Health (NIMH) introduced the Research Domain Criteria (RDoC) project which intends to explicate fundamental biobehavioral dimensions that cut across the current heterogeneous disorder categories in psychiatry (Cuthbert \& Insel, 2013). One major research domain is defined by arousal/ regulatory systems. In this study, we investigate the relationships between the arousal and regulatory systems domain with EEG-beta phenotypes and psychologically defined behavioral dimensions including insomnia, hyperactivity, and attention. This investigation is applied to a large heterogeneous outpatient psychiatric population. The goal of this study is to better understand an objective neurophysiological marker (an EEGphenotype), and its behavioral implications that are shared across disorders and categories as defined by diagnostic systems such as DSM-IV/5 (American Psychiatric Association, American Psychiatric 2013).

The resting state EEG consists of several qualitatively different patterns that can be used to group the variance of the electrophysiological time-series into a small number of categories of phenotypical divergence, as suggested by Johnstone et al. (2005). From this point of view, phenotypes are the intermediate step between genotype and behavior and thus are more closely related to behavior than genotype is related to behavior. While the commonly used terms "biological marker", "trait", "biomarker" etc. refer to differences between patient groups which do not necessarily have a hereditary basis, a 'phenotype' refers to a marker when certain heritability indicators are also fulfilled (Gottesman and Gould (2003). In 2005, Johnstone, Gunkelman and Lunt defined a set of EEG patterns as "EEG phenotypes" when the genetic links were known, and as "candidate EEG phenotypes" when the linkage to genetics remained unknown. These proposed stable states of neurophysiological function are not identical to the DSM groupings, but were suggested to cut across DSM categories and have implications for pharmacological treatment and neurofeedback. One of the candidate phenotypes is spindling excessive beta (SEB), also referred to as beta spindles, which is defined as 'High frequency beta with a spindle morphology, often with an anterior emphasis' (Johnstone et al. 2005). SEB is quite similar to the sub-vigil-beta described by Kubicki and Ascona (1983): 'Sub-vigil beta bursts are a stable feature characterized by a frequency of 25-35 c/sec. frontal dominance and amplitude rarely exceeding $30 \mu \mathrm{V}$. This SEB should not be confused with other known patterns. One of these is the rare (4 in 3,000) 'paroxysmal fast activity' or 'beta band seizure pattern', which most often occurs during non-REM sleep, but also during waking (Stern \& Engel, 2005) and is most often seen in Lennox-Gastaut syndrome (Halasz et al. 2004). The other possibly confusing EEG pattern in this respect is the rare (0.6\%) EEG pattern of 'occipital slow beta waves' also termed 'quick alpha variants 16-19/sec'. This pattern responds in the same way as alpha to eyes opening and also has a similar topographic distribution as described by Vogel (1970).

Family studies have shown that frontal and frontocentral SEB and excess beta exhibit an autosomal dominant mode of inheritance in healthy persons and that there is a lower frequency of fronto-precentral beta in Japanese subjects (Vogel, 1970). A strong linkage between beta frequencies and GABA-A receptor genes has been reported (Porjesz et al., 2002) suggesting this SEB pattern might be considered an EEGphenotype. Besides an EEG-phenotype, SEB has also been suggested to be a state 
marker and was found associated with sub-vigil states that occur during drowsiness and in sleep stage 1 (Kubicki \& Ascona, 1983). Furthermore, SEB is most typically observed as a medication effect due to sedating drugs such as benzodiazepines (Blume 2006) or barbiturates (Schwartz et al. 1971), further supporting SEB as a reflection of sub-vigil states.

Although SEB seem to have no direct diagnostic utility Kozelka \& Pedley (1990); Arns et al., 2008), there is evidence that its presence as an EEG-phenotype or a state marker of drowsiness is linked with psychopathology at the behavioral level. Van Sweden (1986) and colleagues described SEB in 2 unmedicated manic patients who also exhibited profound insomnia and interpreted this to be reflective of reduced vigilance. Similar findings have been reported in other studies where these were referred to as 'periods of microsleep' (Small et al. 1997) or signs of reduced EEG-vigilance (Schoenknecht et al. 2010). Further, in the EEG classification scheme of Davis (1941) and also employed by Finley \& Campbell (1941), five different groupings are defined ranging from 'normal' (group I) to 'abnormal' (group IV) to 'very abnormal' (group V). In this classification, SEB is defined under the group IV heading and EEG records with this fast activity were found more often in manic-depressive cases as in schizophrenia (Finley \& Campbell, 1941). On the other hand, increased beta power is often interpreted as hyperarousal. However, this notion often refers to desynchronized activity in the beta band and not specifically to the synchronized SEB as defined above. Several researchers have also interpreted this beta as a sub-vigil state, hypoarousal state, or 'paradoxical beta activation' (Bente, 1964, p. 154, figure 54, 80; for review see Ulrich (Ulrich 1994, page 161)). Furthermore, Clarke et al. (2013) recently demonstrated that ADHD children with a high Theta-Beta ratio, as well as excess beta, both have low arousal as indexed by skin conductance, further suggesting that this excess beta group is associated with hypoarousal.

There is more compelling evidence for a proposed linkage between SEB and psychopathology coming from Attention Deficit Hyperactivity Disorder (ADHD) research. Here, excess beta can be found in up to $13-20 \%$ of patients (Chabot \& Serfontein, 1996; Clarke et al. 1998, Clarke et al. 2001a), and these patients do respond well to stimulant medication (Chabot et al. 1999; Clarke et al., 2003; Hermens et al. 2005). Arns et al. (2008) found that when specifically looking at frontal SEB, the prevalence of this beta-subtype has been found to be comparable between children with and without ADHD (15-22\%). Additionally, Clarke et al. (2001b) reported this ADHD sub-group was more prone to moody behavior and temper tantrums and this subgroup is also characterized by hypoarousal as measured by skin conductance (Clarke et al., 2013). From clinical practice it is known that when this SEB in ADHD patients is down-trained by neurofeedback, this results in clinical improvement (Arns et al. 2012). For adults with and without depression (Arns 2011), similar rates have also been reported for frontal SEB, supporting the notion that SEB is not of diagnostic value (Kozelka \& Pedley 1990; Johnstone et al. 2005), but could reflect a bio-behavioral dimension that cuts across diagnostic entities such as ADHD and Depression see Figure 1A and 1B for examples of SEB.

In this study we aimed to investigate the relationship between SEB and behavior. In line with the $\mathrm{RDoC}$ proposal, our primary analysis was focused on a heterogeneous psychiatric population in order to determine if this EEG-phenotype is associated with specific behavioral phenomena that cut across DSM categories. We specifically looked at measures of sleep (insomnia and hypersomnia; given earlier reports that SEB could 


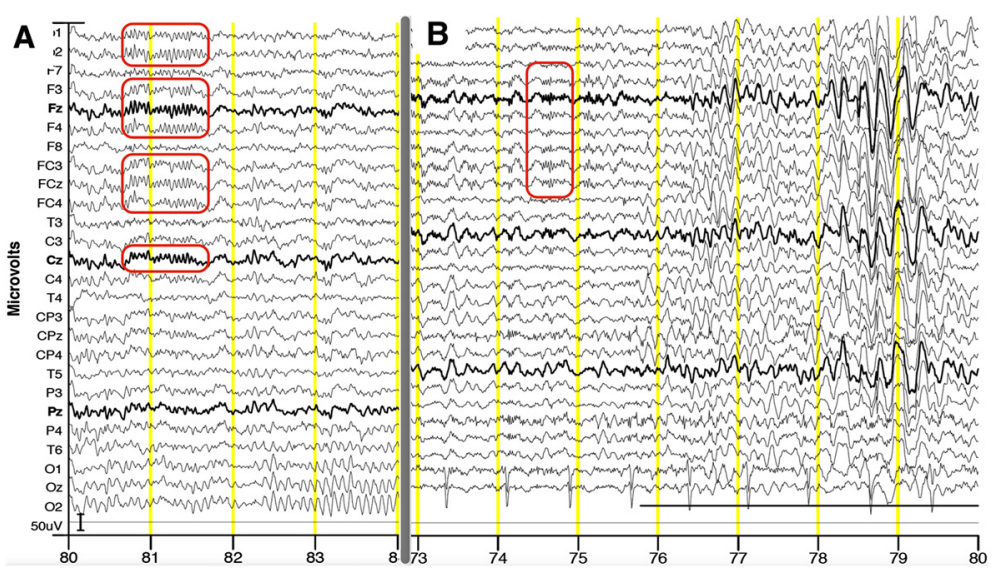

Figure 1 Two clear examples of SEB. A) An EEG with clear frontal SEB, exceeding $20 \mu \mathrm{V}$ peak-peak, B) a paroxysmal EEG, where the same channels with the maximum paroxysmal event (78-80 s.), also show the beta spindling prior to the paroxysm (beta spindles highlighted in the red box). The scale on the $\mathrm{Y}$-axis represents seconds.

be reflective of a sub-vigil state) and measures of inattention and hyperactivity/impulsivity given the earlier reported role of sub-vigil beta and the clear presence of SEB in ADHD. In addition we conducted a secondary analysis on a subgroup diagnosed with ADHD, to further verify if the strength of the association changed when looking within this specific DSM category. Since it is known that SEB can be induced by benzodiazepines and beta-spindles often co-occur with seizure disorders, we excluded those subjects on benzodiazepines and with a history of seizure disorder. We hypothesize that SEB is a) associated with sleep problems, specifically insomnia and b) inattention and hyperactivity/impulsivity complaints.

\section{Methods}

Data was obtained from the Tarnow Center for Self-Management (TCSM) in Houston, TX, a multidisciplinary practice that treats a wide variety of diagnoses, many of which have failed multiple medication trials and this study employed a retrospective chart review. Diagnoses were made by board certified psychiatrists and psychologists according to the DSM-IV-TR criteria. The data was collected over a five-year period for those who were referred for an EEG/qEEG assessment. The EEG/qEEG archival database used at TCSM contains demographic information, diagnosis, EEG phenotypes, and the number of medications prescribed for 427 clinical cases.

In addition, for secondary analysis, a rigidly defined ADHD group was created from this sample where patients needed to have a clinician-confirmed diagnosis of ADHD and have at least 6 symptoms or more on the ADHD-RS items for IA or HI. This secondary screening resulted in a sub-sample of 49 rigidly defined ADHD patients.

This database was submitted to the Texas Southern University's (TSU) Committee for Protection of Human Subjects (CPHS) and was approved to meet the exemption categories set forth by the federal regulation 45 CFR 46.101 (b) (2) and (4).

\section{Questionnaires and psychopathology}

Given our heterogeneous psychiatric population, standard questionnaires such as a Beck's Depression Inventory (BDI) or ADHD rating scale (ADHD-RS) are mostly used 
to index complaints in a sub-group of patients only (i.e. depression and ADHD respectively). Therefore, in this study, we used a generic 300 item-screening questionnaire (CNC1020; EEG Professionals, The Netherlands) designed to assess various psychological aspects. This resulted in standardized data across various DSM disorders and enabled us to link physiology to behavior, independent of DSM diagnosis, and in line with the RDoC approach. Data on construct and predictive validity of this test can be found here (http://www.eegprofessionals.nl/cnc1020_isnr_2011.pdf) and CNC1020 was reported to have high reliability (Cronbach's alpha $=0.982$ ). Under analysis more details are provided on how these items were scored and a score similar to the ADHD-RS-IV and sleep items were obtained.

\section{Electroencephalogram}

The patient's EEG was recorded using the Deymed TruScan 32 equipment (Deymed, Prague), with impedance maintained below $10 \mathrm{k} \mathrm{Ohm}$. The patients were seated in a slightly reclining chair in a silent and low light environment. An "Electrocap" was used to collect the data according to the International 10-20 System with linked ears (Fp1, Fp2, F7, F3, Fz, F4, F8, T3, C3, Cz, C4, T4, T5, P3, Pz, P4, T6, O1, and O2). A minimum of 20 minutes total data was recorded in both eyes open $(10 \mathrm{~min})$ and eyes closed $(10 \mathrm{~min}$.) resting conditions (order of these could vary among subjects). All patients (or parent) were instructed to not take any stimulant medications or drink caffeinated drinks in the morning of the EEG.

All data were manually de-artifacted, processed and analyzed by the same team (RJS \& JG) using both the Human Brain Index (HBI) and the Neuroguide databases as appropriate to the age of the client. The raw EEG and quantitative EEG (QEEG) data were analyzed and reported on a case-by-case basis during routine clinical evaluation of the 427 cases, and not part of a post-hoc analysis of the client database used in this study. Drowsiness was reported when observed; though analysis was restricted to eyes open resting and eyes closed resting, excluding drowsiness and sleep state content from these results.

\section{EEG assessment and classification}

EEGs were rated consistent with the pattern definitions from Johnstone et al. ('High frequency beta with a spindle morphology, often with an anterior emphasis': Johnstone et al. 2005) and the examples labeled as 'Beta Spindles' from Clarke and colleagues (2001b) by author JG. For this study, the focus was on cases with ratings of SEB. The electrode site with maximum power of SEB and peak frequency of SEB was determined by RJS based on visual analysis of the topographical mapping provided in the routine EEG/qEEG reports. In a previous study a high inter-rater reliability of scoring SEB was reported (Kappa: 0.97; $\mathrm{p}<.0001$ ) where authors MA and JG independently scored the occurrence of SEB (Arns et al. 2008), hence in this study we relied on SEB visually rated by JG. Furthermore, from the quantitative EEG (QEEG) reports where data were compared to a normative EEG database (HBI and Neuroguide) it was further verified that the observed SEB was a) indeed in excess based on Z-scores, b) were present primarily in the beta band; c) verified that the sites where this SEB was observed matched the topography of the deviating Z-scores, and d) were in excess compared to age appropriate norms. 


\section{Analysis}

From the 300 item CNC1020, 18 items were extracted that were identical to the ADHD-RS-IV, 9 for inattention (IA) and 9 for hyperactivity and impulsivity (HI). The original ADHD-RS-IV is rated as $0,1,2$, and 3 , therefore the mean score of the $\mathrm{CNC} 1020$ is divided by 8 (max. CNC score), multiplied by 3 (max. score on ADHD-RS-IV), and multiplied by 9 (\# of items). This results in the sum score for IA and $\mathrm{HI}$, which is comparable to other published studies using the ADHD-RS-IV. In addition, the number of IA and HI symptoms have been established by using 4 as a cut-off for the CNC1020 data to denote a symptom is present, resulting in 0-9 symptoms for IA or HI, similar to the scoring for the ADHD-RS-IV.

In addition, 3 items indexing hypersomnia (I sleep too much; I get the recommended amount of sleep but don't feel rested; I am sluggish and have trouble getting going in the morning) and 5 items indexing insomnia were extracted (When I awaken to go to the bathroom, I have trouble falling back to sleep; I am easily awakened by dreams, and have trouble falling back to sleep; I easily awaken to any noise inside or outside my home and have trouble falling back to sleep; I move around a lot in my sleep; I have trouble falling asleep at night). For these an averaged score expressed in a percentage was calculated for hypersomnia and insomnia.

SEB was scored per EEG electrode site (defined as the site with maximum amplitude) and the center frequency of the spindles was established. Frontal SEB was defined as SEB at F3, Fz or F4 (frequency: 12.1\%). Sites with frequencies of SEB less than 5\% were not analyzed due to too low sample sizes for further statistical analyses.

\section{Statistics}

The relationship between IA, HI, and sleep problems, as well as age, was investigated with a one-way ANOVA with factor group (with or without SEB) within the whole population, and also more specifically within a sub-group with ADHD. Differences in gender were tested using a chi-square. Based on significant associations found between SEB and behavior, exploratory analysis was performed on a per item level.

Given the exploratory nature of this study with some repeated testing and large sample size, we have set the significance level to a conservative $\mathrm{p} \leq .01$ (two-tailed). Effect sizes (ES) are reported as Cohen's $d$.

\section{Results}

Data from a total of 427 patients were available for this study. Only patients that were using benzodiazepines $(\mathrm{N}=12)$ and patients with a history of seizures $(\mathrm{N}=25)$ were excluded, resulting in a sample of 390 psychiatric patients $(\mathrm{N}=269$ males; $\mathrm{N}=121$ females; average age $=20.1 \mathrm{yrs}, \mathrm{SD}: 14.7)$. The sample cases included the following DSM diagnoses: Attention Deficit Hyperactivity Disorder (65.4\%), Autistic Spectrum Disorder (22.3\%), Mood Disorders (25.1\%), Anxiety Disorders (39.2\%), Psychotic Disorders (1.8\%), Substance Abuse Disorder (2.3\%), Tourette's Syndrome (6.9\%), and Oppositional Defiant Disorder (3.8\%). It is noted that more than one DSM diagnosis may be present in a single case, thus the total of the diagnostic category percentages is greater than $100 \%$.

Occurrence of SEB per electrode site varied between $0-10.8 \%$. Also see Figure 2 for a topographical plot illustrating the occurrence of SEB per site in percentages where 


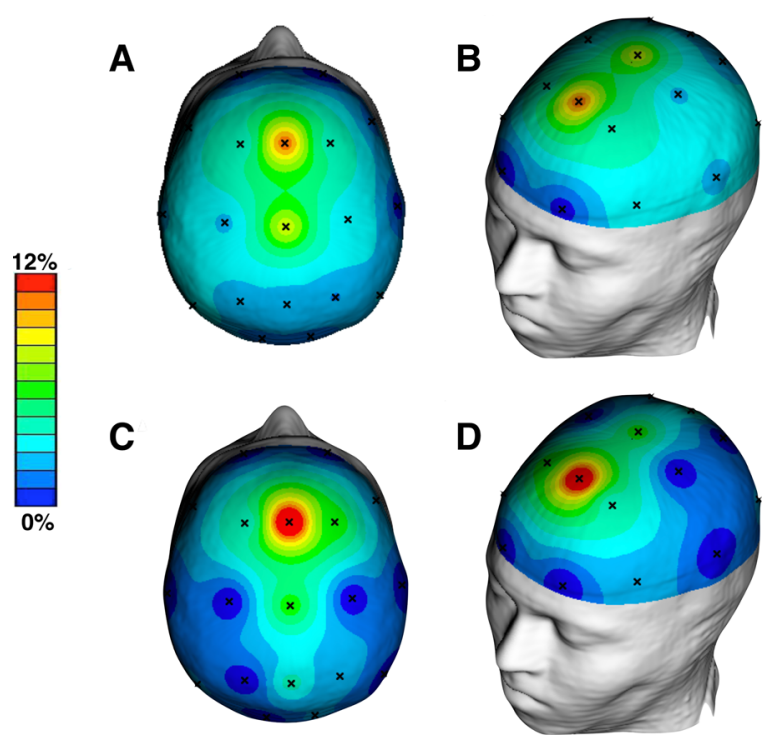

Figure 2 Topographic plots depicting the percentage occurrence of SEB at various cortical locations in a diverse psychiatric population, (top, $A$ and $B$ ) and a sub-group of patients with ADHD only (bottom, C and D): scale for all plots is $0 \%$ (blue) to $12 \%$ (red). The highest occurrence of SEB is found at Fz (Group: 10.8\%; ADHD: 14.3\%) followed by Cz (Group: 7.9\%; ADHD: 6.1\%). Note the similarities in topography, further demonstrating the lack of diagnostic utility for SEB (in line with previous studies e.g. Arns et al., 2008).

frontal and central SEB are most prevalent. For further analyses SEB was grouped into Frontal SEB (F3, Fz, F4: N = 47/390: 12.1\%).

The topographical distribution of the SEB in the rigidly defined ADHD cases $(\mathrm{N}=49)$ is contrasted with the distributions in the 390 diverse clinical cases (see Figure 2). Note the similarities in topography of SEB for both groups.

\section{Whole sample analysis}

For the whole sample of 390 psychiatric patients, 47 (12.1\%) had frontal SEB, and they did not differ from subjects without SEB in age $(\mathrm{p}=.323)$, gender $(\mathrm{p}=.137)$, and number of drugs prescribed $(\mathrm{p}=.107)$. The average SEB frequency was $20.6 \mathrm{~Hz}(\mathrm{SD}=4.1 \mathrm{~Hz}$; range 14-30 Hz). Patients with SEB had more HI symptoms $(\mathrm{p}=.004, \mathrm{~F}=8.214 ; \mathrm{DF}=1,389$ : $\mathrm{ES}=0.41)$ and more insomnia complaints $(\mathrm{p}=.002, \mathrm{~F}=9.762$; $\mathrm{DF}=1,384$; $\mathrm{ES}=0.48)$. Repeating this analysis for SEB at $\mathrm{Cz}$ yielded no differences in IA, HI symptoms, and insomnia. The prevalence of SEB for all other sites was too low $(<5 \%)$ to yield reliable statistics.

Since it is unclear if the above results could indicate that SEB is associated with insomnia, which potentially may cause higher levels of impulsivity and hyperactivity, we conducted an additional analysis. Running an ANCOVA, controlling for age and $\mathrm{HI}$ symptoms, resulted in a significant effect for insomnia ( $\mathrm{p}=.007, \mathrm{~F}=7.486, \mathrm{DF}=1,384$ ); whereas the same analysis, controlling for age and insomnia, resulted in a non-significant main effect for HI symptoms ( $\mathrm{p}=.214$ ), suggesting the relation of SEB to HI symptoms is mediated by insomnia. Also see Figure 3 for a graphical depiction of these results.

Within this group with frontal SEB, no correlations were found between the SEB frequency and HI symptoms $(\mathrm{p}=.902)$ and Insomnia $(\mathrm{p}=.783)$. When excluding subjects with a SEB frequency equal to or below $18 \mathrm{~Hz}(\mathrm{~N}=12)$, the results remained unchanged 


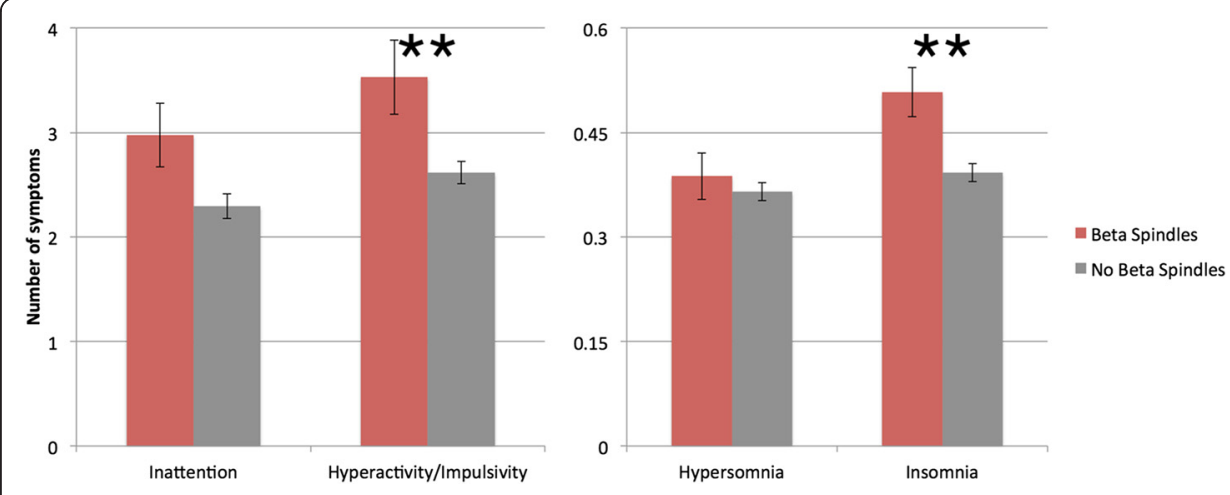

Figure 3 Differences in symptoms between psychiatric patients with and without frontal SEB, where patients with frontal SEB have an increased number of $\mathrm{HI}$ symptoms and increased signs of insomnia $(* *: p<.005)$.

(HI: $\mathrm{p}=.018$; Insomnia: $\mathrm{p}=.007$ ), suggesting these effects are not driven by activity in the sleep spindle frequency of $12-15 \mathrm{~Hz}$. Drowsiness was also excluded as a state by the EEG/qEEG processing being restricted to eyes closed waking and eyes open conditions.

\section{Item level}

Repeating the above analyses on the item level revealed the following significant effects for frontal SEB, where all items indicated higher levels of that item for the SEB group.

\section{Impulse control items:}

- Interrupt others: $(\mathrm{p}=.001 ; \mathrm{ES}=0.51)$

- Blurt out answers to questions before the questions have been completed: ( $\mathrm{p}=.001$; $\mathrm{ES}=0.48)$

- Have difficulty waiting in line or waiting my turn in games or group situations: $(\mathrm{p}=.001 ; \mathrm{ES}=0.45)$

\section{Insomnia items:}

- When I awaken to go to the bathroom, I have trouble falling back to sleep: $(\mathrm{p}=.004$; $\mathrm{ES}=0.43)$

- I am easily awakened by dreams, and have trouble falling back to sleep: $(\mathrm{p}=.003$; $\mathrm{ES}=0.45)$

- I easily awaken to any noise inside or outside my home and have trouble falling back to sleep: $(\mathrm{p}=.001 ; \mathrm{ES}=0.52)$

- I have trouble falling asleep at night: $(\mathrm{p}=.854)$

These findings suggest that frontal SEB in a psychiatric population are specifically associated with impulse control items and sleep maintenance and that the earlier effects are mostly driven by these items, and not as much by hyperactivity and sleep onset problems. 


\section{Discussion}

In this study we found specific associations between the EEG-phenotype "frontal SEB" and impulse control problems as well as insomnia complaints (specifically sleep maintenance problems) as indicated by the CNC1020 questionnaire in an unselected psychiatric population. Interestingly, altered impulse control and complaints of insomnia are symptoms that cut across many diagnostic entities such as ADHD, Depression, Substance Abuse Disorders, etc. and SEB is equally present in healthy controls as in patients with ADHD (Arns et al., 2008) and Depression (Arns 2011), and the topography of SEB is also rather similar for ADHD as compared to a heterogeneous psychiatric population as can be seen in Figure 2 .

The observed associations demonstrated medium efffect sizes (Cohen's $d=0.41-0.48$ ) on the group level. Mediator analyses suggested that the insomnia effects explained the impulse control problems, indicating that the sleep maintenance difficulties mediate the impulse control problems. Therefore, the frontal SEB is most likely also a result of sleep maintenance problems, in line with the notion of sub-vigil beta from Kubicki and Ascona (1983), excess beta to be a reflection of hypoarousal (Clarke et al., 2013) and the occurence of SEB in response to sedating drugs such as benzodiazepines (Blume 2006) and barbiturates (Schwartz et al., 1971).

Interestingly, a recent meta-analysis of fMRI results demonstrated that inhibtion problems in ADHD were specifically associated with reduced activation in the supplementary motor area (SMA), right inferior prefrontal cortex (r-IFC), and the thalamus (Hart et al., 2013). In addition the networks associated with attention exhibited a completely different network with decreased activation in the right dorsolateral prefrontal cortex (rDLPFC), the left putamen and globus pallidus, the pulvinar and caudate tail, and increased activation in the left cuneus and right cerebellum. Our results tend to be consistent with these findings, given that the SMA area is localized between electrode sites $\mathrm{Fz}$ and $\mathrm{Cz}$ (see Figure 2 for matching localization of SEB), and we only found these effects for impulse control specifically, though not for inattention. The midline dominance of frontal SEB is also further suggestive of a medial origin of this SEB, and the involvement of the thalamus as demonstrated by Hart et al. (2013) suggests this SEB could be reflective of a thalamo-cortical network.

Future studies should use inverse solutions such as LORETA and dipole analysis to further localize SEB, and identify which specific region of interest (ROI) is involved with this association, thereby aiding in a better understanding of this relationship and confirming these originate from the same networks as described by Hart et al. (2013) In addition future studies could also employ techniques such as neurofeedback to downtrain this SEB, and simultanously assess sleep and impulse control to specifically investigate which resolves first, in order to investigate the causality between these domains further. Furthermore, future studies could also combine analyses indexing EEG Vigilance using the VIGALL algorithm to further substantiate SEBs occuring more likely in low vigilance stages. Finally, future studies should more specifically investigate neuropsychological tasks measuring aspects of inattention and impulse control seperately, to further demonstrate the specificity of these effects. Limitations of the study include the use of a non-validated sleep-questionnaire and patients were not free of medication at baseline. 


\section{Conclusion}

The SEB we observed is unlikely to be related to classical $12-15 \mathrm{~Hz}$ sleep spindles. As can be seen in Figure 2, the site with the highest occurrence of SEB was Fz, whereas sleep spindles are most often found more centrally and at lateralized sites. Additionally, the analysis and processing of the EEG quantitatively, specifically excluded drowsiness. Furthermore, SEB frequency did not correlate with insomnia and HI complaints, and when excluding SEB that overlapped with the characteristic sleep spindle frequency $(12-15 \mathrm{~Hz})$, the results remained unchanged.

In addition, it is known that a majority of ADHD patients (70-80\%) suffer from sleep onset issues, also called sleep onset-insomnia (Van der Heijden et al. 2005; Van Veen et al. 2010; Arns et al. 2014 reviewed in (Arns \& Kenemans, 2014) that could explain specifically inattention problems. In this study the item 'I have trouble falling asleep' was not associated at all with the frontal SEB, but only items related to sleep maintenance. This suggests that the SEB subgroup may be a distinct and specific sub-group of ADHD and psychiatric patients, with a specific etiology associated with sleep maintenance problems and impulsivity.

An additional question is whether frontal SEB is a true endophenotype causing sleep maintenance and impulse control problems, or is SEB an EEG reflection of sleep maintenance problems? It is known that sleep problems and fatigue manifest themselves in the EEG as excess frontal theta or frontal alpha (Arns \& Kenemans, 2014). A strong linkage between beta frequencies and GABA-A receptor genes has been reported (Porjesz et al., 2002). Hypnotic drugs such as benzodiazepines (Blume 2006) and barbiturates (Schwartz et al., 1971) have been found to be iatrogenic for SEB. Additionally, earlier mentioned studies interpreting SEB as sub-vigil beta or hypoarousal suggests that $\mathrm{SEB}$ reflects signs of disturbed vigilant regulation. Therefore, these findings suggest a specific pathway of sleep maintenance problems resulting in frontal SEB (in line with the sub-vigil beta as described in the introduction) and in impulse control problems, maybe positing SEB more as a state rather then a trait (EEG Phenotype) marker, however this should be investigated in more detail.

Competing interests

MA reports research grants and options from Brain Resource Ltd. (Sydney, Australia), acted as a paid consultant for the United BioSource Corporation (UBC), Bracket, Mindmedia and Vivatech and is a co-inventor on 3 patent applications (A61B5/0402; US2007/0299323, A1; WO2010/139361 A1) related to EEG, neuromodulation and psychophysiology, but does not own these nor receives any proceeds related to these patents. SO, RJS and JG report no conflicts of interest.

Authors' contributions

RS initiated this manuscript, collected all the data in his clinic and scored all data, MA performed all statistical tests and wrote the first draft of the manuscript, JG rated all EEGs and SO, and the other authors all contributed to writing up the manuscript. All authors read and approved the final manuscript.

Acknowledgement

We want to thank Judy Crawford for proofreading of an earlier version of the manuscript.

\section{Author details}

${ }^{1}$ Department of Experimental Psychology, Utrecht University, Utrecht, The Netherlands. ${ }^{2}$ Research Institute Brainclinics, Bijleveldsingel 34, Nijmegen 6524 AD, The Netherlands. ${ }^{3}$ The Tarnow Center for Self-Management, Houston, Texas, USA. ${ }^{4}$ Brain Science International, San Ramon, California, USA. ${ }^{5}$ Clinic for Psychiatry and Psychotherapy, University Hospital, Leipzig, Germany. 
References

American Psychiatric Association (2013) Diagnostic and statistical manual of mental disorders, 5th edn. American Psychiatric Association, Washington, DC

Arns M (2011) Personalized medicine in ADHD and depression: a quest for EEG treatment predictors. PhD Thesis, Utrecht University.

Arns M, Feddema I, Kenemans JL (2014) Differential effects of theta/beta and SMR neurofeedback in ADHD on sleep onset latency. Front Hum Neurosci 8:1019, doi:10.3389/fnhum.2014.01019

Arns M, Kenemans $J$ (2014) Neurofeedback in ADHD and insomnia: Vigilance stabilization through sleep spindles and circadian networks. Neuroscience and Biobehavioral Reviews 44:183-194, doi:10.1016/j.neubiorev.2012.10.006

Arns M, Drinkenburg W, Kenemans JL (2012) The effects of QEEG-informed neurofeedback in ADHD: an open-label pilot study. Appl Psychophysiol Biofeedback 37(3):171-80, doi:10.1007/s10484-012-9191-4

Arns M, Gunkelman J, Breteler M, Spronk D (2008) EEG phenotypes predict treatment outcome to stimulants in children with ADHD. J Integr Neurosci 7(3):421-38

Bente, D (1964) Thesis. Retrieved from google scholar.

Blume WT (2006) Drug effects on EEG. J Clin Neurophysiol 23(4):306

Chabot RJ, Serfontein G (1996) Quantitative electroencephalographic profiles of children with attention deficit disorder. Biol Psychiatry 40(10):951-63, doi:10.1016/0006-3223 (95) 00576-5

Chabot RJ, Orgill AA, Crawford G, Harris MJ, Serfontein G (1999) Behavioral and electrophysiologic predictors of treatment response to stimulants in children with attention disorders. J Child Neurol 14(6):343-351, doi:10.1177/088307389901400601

Clarke AR, Barry RJ, McCarthy R, Selikowitz M (1998) EEG analysis in attention-deficit/hyperactivity disorder: a comparative study of two subtypes. Psychiatry Res 81(1):19-29

Clarke AR, Barry RJ, McCarthy R, Selikowitz M (2001a) EEG-defined subtypes of children with attention-deficit/ hyperactivity disorder. Clin Neurophysiol 112(11):2098-2105

Clarke AR, Barry RJ, McCarthy R, Selikowitz M (2001b) Excess beta activity in children with attention-deficit/hyperactivity disorder: an atypical electrophysiological group. Psychiatry Res 103(2-3):205-18

Clarke AR, Barry RJ, McCarthy R, Selikowitz M, Clarke DC, Croft RJ (2003) Effects of stimulant medications on children with attention-deficit/hyperactivity disorder and excessive beta activity in their EEG. Clin Neurophysiol 114(9):1729-37

Clarke AR, Barry RJ, Dupuy FE, McCarthy R, Selikowitz M, Johnstone SJ (2013) Excess beta activity in the EEG of children with attention-deficit/hyperactivity disorder: a disorder of arousal? Int J Psychophysiol 89(3):314-9, doi:10.1016/ j.ijpsycho.2013.04.009

Cuthbert BN, Insel TR (2013) Toward the future of psychiatric diagnosis: the seven pillars of rdoc. BMC Med 11:126, doi:10.1186/1741-7015-11-126

Davis PA (1941) Electroencephalograms of manic-depressive patients. American Journal of Psychiatry 98(3):430

Finley KH, Campbell CM (1941) Electroencephalography in schizophrenia. The American Journal of Psychiatry 98(3):374-381

Gottesman II, Gould TD (2003) The endophenotype concept in psychiatry: Etymology and strategic intentions. The American Journal of Psychiatry 160(4):636-45

Halasz P, Janszky J, Barcs G, Szcs A (2004) Generalised paroxysmal fast activity (GPFA) is not always a sign of malignant epileptic encephalopathy. Seizure: J British Epilepsy Association 13(4):270-276

Hart H, Radua J, Nakao T, Mataix-Cols D, Rubia K (2013) Meta-analysis of functional magnetic resonance imaging studies of inhibition and attention in attention-deficit/hyperactivity disorder: exploring task-specific, stimulant medication, and age effects. JAMA Psychiatry 70(2):185-98, doi:10.1001/jamapsychiatry.2013.277

Hermens DF, Cooper NJ, Kohn M, Clarke S, Gordon E (2005) Predicting stimulant medication response in ADHD: evidence from an integrated profile of neuropsychological, psychophysiological and clinical factors. J Integr Neurosci 4(1):107-21

Johnstone J, Gunkelman J, Lunt J (2005) Clinical database development: characterization of EEG phenotypes. Clinical EEG and Neuroscience: Official Journal of the EEG and Clinical Neuroscience Society (ENCS) 36(2):99-107

Kozelka JW, Pedley TA (1990) Beta and mu rhythms. J Clin Neurophysiol: Official Publication of the American Electroencephalographic Society 7(2):191-207

Kubicki S, Ascona A (1983) Genetic basis of beta patterns of the EEG in the adult. Rev Electroencephalogr Neurophysiol Clin 13(3):245-52

Porjesz B, Almasy L, Edenberg HJ, Wang K, Chorlian DB, Foroud T, Begleiter H (2002) Linkage disequilibrium between the beta frequency of the human EEG and a GABAA receptor gene locus. Proc Natl Acad Sci U S A 99(6):3729-33, doi:10.1073/pnas.052716399

Schoenknecht P, Olbrich S, Sander C, Spindler P, Hegerl U (2010) Treatment of acute mania with modafinil monotherapy. Biological Psychiatry 67. doi:10.1016/j.biopsych.2009.12.021

Schwartz J, Feldstein S, Fink M, Shapiro DM, Itil TM (1971) Evidence for a characteristic EEG frequency response to thiopental. Electroencephalogr Clin Neurophysiol 31(2):149-53

Small JG, Milstein V, Medlock CE (1997) Clinical EEG findings in mania. Clinical EEG (electroencephalography) 28(4):229-35

Stern JM, Engel J (2005) Atlas of EEG patterns. Lippincott Williams \& Wilkins, Philadelphia

Ulrich G (1994) Psychiatrische elektroenzephalographie. Jena-Stuttgart-New York: Gustav Fischer Verlag.

Van der Heijden KB, Smits MG, Van Someren EJW, Gunning WB (2005) Idiopathic chronic sleep onset insomnia in attentiondeficit/hyperactivity disorder: a circadian rhythm sleep disorder. Chronobiol Int 22(3):559-70, doi:10.1081/CBI-200062410

Van Sweden B (1986) Disturbed vigilance in mania. Biol Psychiatry 21(3):311-3

Van Veen MM, Kooij JJS, Boonstra AM, Gordijn MCM, Van Someren EJW (2010) Delayed circadian rhythm in adults with attention-deficit/hyperactivity disorder and chronic sleep-onset insomnia. Biol Psychiatry 67(11):1091-6, doi:10.1016/j.biopsych.2009.12.032

Vogel F (1970) The genetic basis of the normal human electroencephalogram (EEG). Human Genetics 10(2):91-114 